\title{
Numerical justification of Leonov conjecture on Lyapunov dimension of Rossler attractor
}

\author{
Kuznetsov N.V., Mokaev T.N., Vasilyev P.A. \\ Draft $^{1}$
}

\begin{abstract}
Exact Lyapunov dimension of attractors of many classical chaotic systems (such as Lorenz, Henon, and Chirikov systems) is obtained. While exact Lyapunov dimension for Rössler system is not known, G.A. Leonov formulated the following conjecture: Lyapunov dimension of Rössler attractor is equal to local Lyapunov dimension in one of its stationary points. In the present work Leonov's conjecture on Lyapunov dimension of various Rössler systems with standard parameters is checked numerically.
\end{abstract}

Keywords: Rössler system, Lyapunov dimension, strange attractor, self-excited and hidden attractor, Lyapunov exponent, chaos, Perron effects, Leonov's conjecture

\section{Introduction}

Lyapunov exponents (LEs) play an important role in the description of dynamical systems behavior. They were introduced by A.M. Lyapunov Lyapunov $|1892|$ for the analysis of stability by the first approximation for regular time-varying linearizations, where the negativeness of the largest Lyapunov exponent indicates stability. Much later, in 1940s, N.G. Chetaev tried to prove that for regular time-varying linearizations, a positive Lyapunov exponent indicates instability in the sense of Lyapunov, but a gap in his proof was revealed and filled recently for more weak definition of instability Leonov and Kuznetsov |2007|). Since there are no general methods for checking regularity of linearization and there are known Perron effects Kuznetsov and Leonov |2005a:bc|; Leonov and Kuznetsov |2007| of sign inversion of the largest Lyapunov exponent for nonregular time-varying linearizations, the computation of Lyapunov exponents for linearization of nonlinear autonomous system along nonstationary trajectories is widely used for investigation of chaos. In this case the positiveness of the largest Lyapunov exponent is often regarded as the indication of chaotic behavior in the considered nonlinear system. The various methods, used for the numerical computation of Lyapunov exponents, are described, e.g., in Benettin et al. |1980a:b|; Shimada and Nagashima |1979|; Wolf et al. |1985|.

Nowadays various characteristics of attractors of dynamical systems (information dimension, metric entropy etc) are studied based on Lyapunov exponents computation. In particular, J.L. Kaplan and J.A. Yorke defined a quantity they called Lyapunov dimension and conjectured that it was equal to information dimension Kaplan and Yorke [1979|.

In the work Leonov $|2012|$ G.A. Leonov considered exact formulas of Lyapunov dimension of Lorenz, Henon, and Chirikov attractors. By analogy with the results for these attractors he conjectured that Lyapunov dimension of Rössler attractor ${ }^{2}$ is determined by a stationary point belonging to this attractor.

In the present paper Leonov's conjecture is checked numerically and it is demonstrated that this conjecture is true for three different types of Rössler systems. These three-dimensional systems are simplest and, in a sense, minimal models for continuous-time chaos. They have only a single nonlinear quadratic term and they

\footnotetext{
${ }^{1}$ PDF slides http://www.math.spbu.ru/user/nk/PDF/Lyapunov-exponent-Sign-inversion-Perron-effects-Chaos.pdf

${ }^{2}$ Following Broer et al. |1991|; Leonov |2008|, an attractor is a bounded, closed, invariant, attracting subset of phase space of dynamical system. Since for the considered Rössler systems there are no analytical estimations of localization of their attractors, it is not feasible to check their boundness and closedness. Usually by Rössler attractor one means an attracting set obtained as a result of numerical experiments Rossler $[1976,1979$.
} 
generate chaotic attractors with a single "leaf" (in contrast to Lorenz attractor). Rössler systems arose as simplified prototypes of some chemical reactions while Otto Rössler researched different types of chaos in chemical kinetics.

\section{Problem statement}

\subsection{Rössler systems}

Consider the following three-dimensional Rössler systems Rossler |1976, 1979]

$$
\text { (1.1) }\left\{\begin{array} { l } 
{ \dot { u } = - y - z } \\
{ \dot { y } = u } \\
{ \dot { z } = a ( y - y ^ { 2 } ) - b z }
\end{array} \quad \text { (1.2) } \left\{\begin{array} { l } 
{ \dot { u } = - y - z } \\
{ \dot { y } = u + a y } \\
{ \dot { z } = b - c z + u z }
\end{array} \quad \text { (1.3) } \left\{\begin{array}{l}
\dot{u}=-y-z \\
\dot{y}=u+a y \\
\dot{z}=b u-c z+u z
\end{array}\right.\right.\right.
$$

with the corresponding standard parameters

$$
\begin{aligned}
& (1.1): a=0,386 ; b=0,2 \\
& (1.2): a=0,2 ; b=0,2 ; c=5,7 \\
& (1.3): a=0,36 ; b=0,4 ; c=4,5 .
\end{aligned}
$$

In the phase spaces of these systems, for parameters (2.2) there exist chaotic attractors and the corresponding stationary points

$$
\begin{aligned}
& x_{0}=(0,0,0) \text { for systems }(1.1) \text { and }(1.3), \\
& x_{0}=\left(\frac{c-\sqrt{c^{2}-4 a b}}{2},-\frac{c-\sqrt{c^{2}-4 a b}}{2 a}, \frac{c-\sqrt{c^{2}-4 a b}}{2 a}\right) \text { for system (1.2) }
\end{aligned}
$$

are located in the middle of these attractors Rossler |1976, 1979|.

\subsection{Lyapunov dimension}

Consider a topological characteristic - a local Lyapunov dimension of the point $x_{0}$ in the phase space $U$ of dynamical system, which is associated with the Lyapunov spectrum $\lambda_{1}\left(x_{0}\right) \geq \ldots \geq \lambda_{n}\left(x_{0}\right)$ and is defined by formula

$$
\operatorname{dim}_{L} x_{0}=j+\frac{\lambda_{1}\left(x_{0}\right)+\ldots+\lambda_{j}\left(x_{0}\right)}{\left|\lambda_{j+1}\left(x_{0}\right)\right|} .
$$

Here $j \in[1, n]$ is the smallest natural number $m$ such that

$$
\lambda_{1}\left(x_{0}\right)+\ldots+\lambda_{m+1}\left(x_{0}\right)<0, \quad \lambda_{m+1}\left(x_{0}\right)<0, \quad \frac{\lambda_{1}\left(x_{0}\right)+\ldots+\lambda_{m}\left(x_{0}\right)}{\left|\lambda_{m+1}\left(x_{0}\right)\right|}<1 .
$$

Lyapunov dimension of invariant set $B \subset U$ of dynamical system is defined by the relation

$$
\operatorname{dim}_{L} B=\sup _{x \in B} \operatorname{dim}_{L} x .
$$

The properties of Lyapunov dimension are considered in details in the works Pesin |1988|; Temam [1993]; Boichenko et al. [2005|. In particular, it is proved that Lyapunov dimension is an upper bound for Hausdorff and fractal dimensions. 


\subsection{Leonov's conjecture}

For Lorenz, Henon, and Chirikov systems a problem of computation of Lyapunov dimension of their attractors is solved in Leonov and Lyashko |1997|; Leonov [1998|; Boichenko et al. |1998|; Boichenko and Leonov |2000|; Leonov et al. $|2011 \mathrm{a}, \mathrm{b}|$. In these works it is obtained analytically exact Lyapunov dimension of attractors of these systems and in Leonov $|2012|$ it is given estimates of Lyapunov dimension of attractor of Rössler system (1.1). Based on these results, G.A. Leonov formulated the following

Conjecture. If a stationary point $x_{0}$ is embedded in attractor $A$ of Rössler systems (2.1), then

$$
\operatorname{dim}_{L} A=\operatorname{dim}_{L} x_{0}
$$

In order to verify this conjecture for attractors of systems (2.1) with parameters (2.2) and stationary points (2.3), in the present work it is developed a special numerical procedure described below. Note that this procedure can be applied similarly to various modifications of Rössler system of higher orders (see, e.g., Rossler |1979|; Szczepaniak and Macek |2008|; Li |2008|).

\section{Numerical justification of Leonov's conjecture}

\subsection{Lyapunov spectrum computation algorithm}

To verify the conjecture, it is used an approach to the computation of Lyapunov spectrum, suggested in the works Benettin et al. |1980a b|. In Wolf et al. |1985| this approach was adapted to computer realization. This method is an iterative process and is a variation of standard QR algorithm for computation of eigenvalues and eigenvectors Golub and van Loan |1996|. It is based on the following definitions and statements.

Consider system (2.1) in general form

$$
\dot{x}=F(x),
$$

where $x(t) \in \mathbb{R}^{n}$ for any $t \in \mathbb{R}, F: U \rightarrow \mathbb{R}^{n}$ is $C^{r}$-smooth function $(r \geq 1)$ on the open set $U \subset \mathbb{R}^{n}$.

Denote by $A(t)=T_{x} F\left(f\left(t, x_{0}\right)\right)$ the Jacobian matrix of system (3.6), where $f\left(t, x_{0}\right)$ is a solution of system (3.6).

Consider two close points $x_{0}$ and $\left(x_{0}+u_{0}\right)$ in the phase space $U$, where $u_{0}$ is a small disturbance of the point $x_{0}$. Then the evolution of vector $u(t)=f\left(t, x_{0}+u_{0}\right)-f\left(t, x_{0}\right)$ can be studied Parker and Chua |1989| by the following linearized system

$$
\dot{u}=A(t) u \text {. }
$$

The solution of equation (3.7) can be represented as $u(t)=\Phi(t) u_{0}$, where $\Phi(t)=T_{x_{0}} f\left(t, x_{0}\right)$ is a fundamental matrix of system (3.7). The exponential rate of divergence (or convergence) of nearby trajectories is given by formula

$$
\lambda\left(x_{0}, u_{0}\right):=\varlimsup_{t \rightarrow \infty} \frac{1}{t} \ln \frac{\|u(t)\|}{\left\|u_{0}\right\|}=\varlimsup_{t \rightarrow \infty} \frac{1}{t} \ln \left\|\Phi(t) u_{0}\right\| .
$$

This value is called Lyapunov exponent of order 1 (or, simply, Lyapunov exponent).

It can be considered a generalization of Lyapunov exponent of order 1 to the case of order $p, 1 \leq p \leq n$. Let $E_{0}^{p}$ be the $p$-dimensional subspace of tangent space $E_{0}$ and $U_{0}$ be the open parallelepiped generated by $p$ linearly independent vectors $e_{1}, \ldots, e_{p}$ of $E_{0}^{p}$. Then Lyapunov exponent of order $p$ is defined Benettin et al. |1980a| as

$$
\lambda^{p}\left(x_{0}, E_{0}^{p}\right):=\varlimsup_{t \rightarrow \infty} \frac{1}{t} \ln \operatorname{Vol}^{p}\left(T_{x_{0}} f\left(t, U_{0}\right)\right)=\varlimsup_{t \rightarrow \infty} \frac{1}{t} \ln \operatorname{Vol}^{p}\left[\Phi(t) e_{1}, \ldots, \Phi(t) e_{p}\right],
$$

where $\mathrm{Vol}^{p}$ means $p$-dimensional volume induced in tangent space by scalar product.

If in (3.8), (3.9) $\varlimsup_{t \rightarrow \infty}$ can be replaced by $\lim _{t \rightarrow \infty}$, then it is said that exact Lyapunov exponent exists. 
It is known Lyapunov |1892|; Oseledec |1968|; Benettin et al. |1980a| that for regular linear systems there exist exact Lyapunov exponents ${ }^{3}$ of order $p, 1 \leq p \leq n$ and in the tangent space $E_{0}$ at the point $x_{0}$ it can be chosen $p$ linearly independent vectors $e_{1}, \ldots, e_{p}$ such that

$$
\lambda^{p}\left(x_{0}, E_{0}^{p}\right)=\lambda_{1}\left(x_{0}\right)+\cdots+\lambda_{p}\left(x_{0}\right),
$$

where $\lambda_{i}\left(x_{0}\right):=\lambda\left(x_{0}, e_{i}\right), i=1 \ldots p$, and $\lambda_{1}\left(x_{0}\right) \geq \ldots \geq \lambda_{p}\left(x_{0}\right)$. That is, each Lyapunov exponent of order $p$ is equal to the sum of $p$ largest Lyapunov exponents of order 1.

In order to calculate all tangent vectors one can solve system (3.6) together with the matrix-valued variational equation Parker and Chua $|1989|$

$$
\dot{\Phi}_{t}\left(x_{0}\right)=A(t) \Phi_{t}\left(x_{0}\right), \quad \Phi_{0}\left(x_{0}\right)=I
$$

where $\Phi_{t}\left(x_{0}\right)=T_{x_{0}} f\left(t, x_{0}\right)$ and $I$ is identity matrix.

In this case one can go directly to the description of computation procedure. Choose the initial point $x_{0}$ and $(n \times n)$ matrix of orthonormal vectors $Q_{0}=\left[q_{1}^{0}, \ldots, q_{n}^{0}\right]$. During the $\mathrm{k}$-th iteration, original system (3.6) is integrated together with variational equation (3.11) with the initial data $\left\{x_{k-1}, Q_{k-1}\right\}$ over the chosen small time interval $h$ for obtaining $x_{k}=f\left(h k, x_{0}\right)$ and

$$
U_{k}=\left[u_{1}^{k}, \ldots, u_{n}^{k}\right]=\Phi_{h k}\left(x_{0}\right) .
$$

Then the matrix $U_{k}$ is $\mathrm{QR}$ decomposed, i.e. $U_{k}=Q_{k} R_{k}$, where $Q_{k}$ is orthogonal matrix and $R_{k}$ is upper triangular matrix. The p-dimensional volume, defined in (3.9), increases by the multiplier $R_{k}(1,1) \cdots R_{k}(p, p)$ since $\mathrm{V}^{p}\left\{u_{1}^{k}, \ldots, u_{p}^{k}\right\}=R_{k}(1,1) \cdots R_{k}(p, p)$, where $R_{k}(i, i)$ is a norm of the vector $u_{i}^{k}, i=1 \ldots p$. The matrix $Q_{k}$ is taken as the initial datum for variational equation at the following iteration.

So, formula (3.9) can be expressed as

$$
\lambda^{p}\left(x_{0}, U_{0}\right)=\lim _{k \rightarrow \infty} \frac{1}{k h} \sum_{i=1}^{k} \ln \left(R_{i}(1,1) \cdots R_{i}(p, p)\right), \quad 1 \leq p \leq n .
$$

One repeats this iteration procedure $K$ times. Subtracting $\lambda^{p-1}$ from $\lambda^{p}$ and using formula (3.10), one obtains approximate values of p-th Lyapunov exponent of order 1 for the chosen trajectory. By formula (2.4) a local Lyapunov dimension can also be computed.

\subsection{Discussion and results}

The algorithm, described in the previous section, is used in the process of justification of Leonov's conjecture. The entire computational procedure is implemented in MATLAB. For the orthogonalization of fundamental matrix it is used MATLAB library function $q r$, which implements a factorization procedure by using the Householder transformation since a classical Gram-Schmidt algorithm is numerically unstable and its modified version requires more execution time.

For nonlinear systems (2.1) there are no exact formulas, describing the solutions of these systems in general form. In this case it is considered approximated solutions, obtained by numerical integration of this systems, which is based on various finite-difference and more complex methods Yan and Ruan |2000|; Al-Sawalha and Noorani $|2009|$. For Rössler system (1.2) the problem of analysis of its analytical and numerical solutions is considered in Letellier et al. |2004|.

In this paper for the integration of systems (2.1) it is used MATLAB realization (solver ode45) of RungeKutta finite-difference schemes of order 4-5 with an adaptive step. The absolute and relative tolerance are

3 The opposite is not true: in the general case the existence of exact Lyapunov exponents does not imply regularity of the system Leonov and Kuznetsov |2007|. 
chosen equal to $10^{-8}$ since smaller values strongly influence a time of evaluation procedure. The parameter of procedure $h$, which determines integration time at each iteration, is chosen sufficiently small for the columns of fundamental matrix to be remained linearly independent. The parameter $K$ - a number of iterations must be sufficiently large in order that the trajectory, with the initial point in the neighborhood of attractor, covered this attractor. For the chosen parameters it was made the following: the number of iterations was increased by 2 times and a step was decreased by 2 times, in which case the result was qualitatively the same.

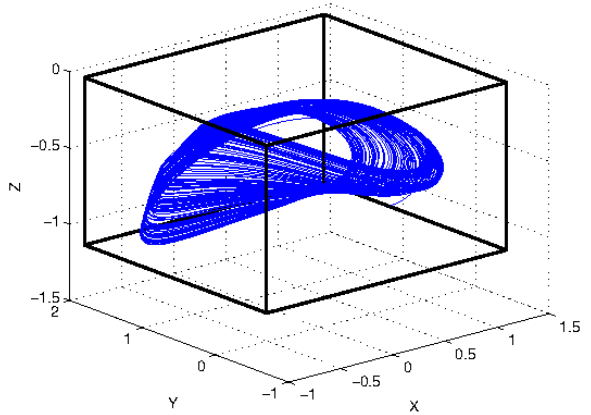

(a) Attractor localization for (1.1)

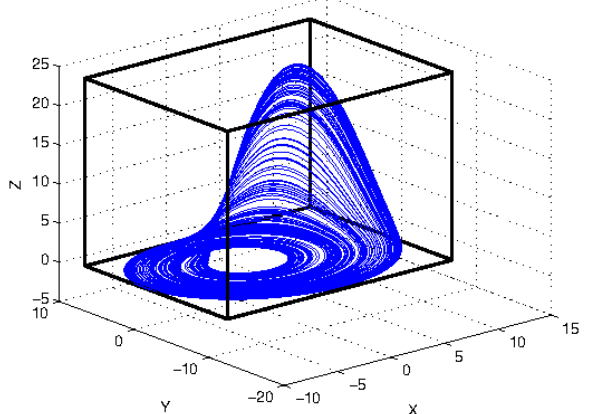

(b) Attractor localization for (1.2)

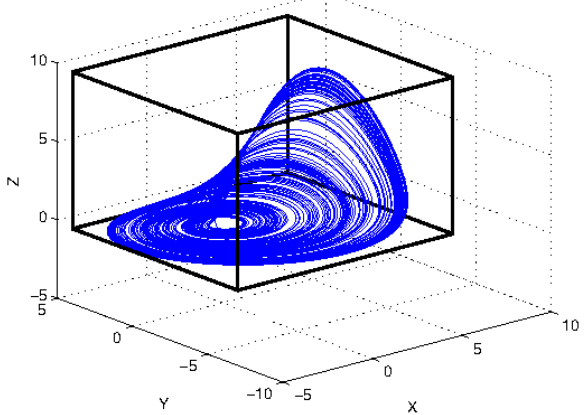

(c) Attractor localization for (1.3)

Figure 1: Localization of attractors of systems (2.1)

Since for Rössler systems (2.1) there are no analytical estimations of localization of their attractors, for estimation it is used computer experiments Barrio et al. $|2009,2011|$. For the considered systems (2.1) their attractors are numerically localized in cubes (Fig. 1) by standard computational procedure ${ }^{4}$. On each cube it is chosen a grid with a certain step and at each grid point it is started the algorithm of computation of local Lyapunov dimension ${ }^{5}$. The obtained values are compared with a local Lyapunov dimension at stationary point. Then it is considered the grid points having the values of Lyapunov dimension, which are most close to a value at stationary point. Around each of these grid points it is considered a grid with a smaller step and at the points of this grid it is computed local Lyapunov dimensions. These values are also compared with a value at stationary point.

Table 1: The results of justification for the following parameters: $h=1, \quad K=200$, abs_tol $=$ rel_tol $=10^{-8}$.

\begin{tabular}{|c|c|c|c|c|}
\hline $\begin{array}{c}\text { Rössler } \\
\text { system }\end{array}$ & Cube & $\begin{array}{c}\text { Grid } \\
\text { step }\end{array}$ & $\max _{x \in \text { grid }} \operatorname{dim}_{L} x$ & $\operatorname{dim}_{L} x_{0}$ \\
\hline$(1.1)$ & {$[-1 ; 1,3] \times[-0,7 ; 1,8] \times[-1,05 ;-0,03]$} & 0,1 & 2,4205 & 2,6042 \\
\hline$(1.2)$ & {$[-9 ; 12] \times[-11 ; 8] \times[-0,1 ; 23,9]$} & 0,5 & 2,0296 & 2,0341 \\
\hline$(1.3)$ & {$[-5 ; 7] \times[-7 ; 4] \times[-0,2 ; 9,8]$} & 0,5 & 2,0340 & 2,0620 \\
\hline
\end{tabular}

\footnotetext{
${ }^{4}$ From a computational point of view, in nonlinear dynamical systems, attractors can be regarded as self-excited and hidden attractors Leonov et al. 2011c|; Bragin et al. |2011|; Leonov et al. 2012 ; Leonov G. A. |2013|. Self-excited attractors can be localized numerically by standard computational procedure, in which after a transient process a trajectory, started from a point of unstable manifold in a neighborhood of equilibrium, reaches a state of oscillation and therefore it can easily be identified. In contrast, for a hidden attractor, its basin of attraction does not intersect with small neighborhoods of equilibria. While many classical attractors are self-exited attractors and therefore can be obtained numerically by standard computational procedure, for localization of hidden attractors it is necessary to develop special procedures since there are no similar transient processes leading to such attractors.

${ }^{5}$ Since numerical localization of attractors is considered and there is no effective way to prove ergodicity rigourously, one has to consider a mesh of initial conditions for investigation of Lyapunov exponents.
} 


\section{Conclusion}

In this work Leonov's conjecture on Lyapunov dimension of various Rössler systems with standard parameters is verified numerically. While the data, given in Table (1), numerically confirm Leonov's conjecture, analytical proof of Leonov's conjecture is still an open problem.

\section{References}

Lyapunov, A.M.. The General Problem of the Stability of Motion. Kharkov; 1892.

Leonov, G.A., Kuznetsov, N.V.. Time-varying linearization and the Perron effects. International Journal of Bifurcation and Chaos 2007;17(4):1079-1107. doi:10.1142/S0218127407017732.

Kuznetsov, N.V., Leonov, G.A.. Criterion of stability to first approximation of nonlinear discrete systems. Vestnik StPetersburg University Mathematics 2005a;38(2):52-60.

Kuznetsov, N.V., Leonov, G.A.. Criteria of stability by the first approximation for discrete nonlinear systems. Vestnik StPetersburg University Mathematics 2005b;38(3):21-30.

Kuznetsov, N.V., Leonov, G.A.. On stability by the first approximation for discrete systems. 2005 International Conference on Physics and Control, PhysCon 2005 2005c;Proceedings Volume 2005:596-599. doi:10.1109/PHYCON . 2005.1514053.

Benettin, G., Galgani, L., Giorgilli, A., Strelcyn, J.M.. Lyapunov characteristic exponents for smooth dynamical systems and for hamiltonian systems. A method for computing all of them. Part 1: Theory. Meccanica 1980a;15(1):9-20.

Benettin, G., Galgani, L., Giorgilli, A., Strelcyn, J.M.. Lyapunov characteristic exponents for smooth dynamical systems and for hamiltonian systems. A method for computing all of them. Part 2: Numerical application. Meccanica 1980b;15(1):21-30.

Shimada, I., Nagashima, T.. A numerical approach to ergodic problem of dissipative dynamical systems. Progress of Theoretical Physics 1979;61(6):1605-1616.

Wolf, A., Swift, J.B., Swinney, H.L., Vastano, J.A.. Determining Lyapunov exponents from a time series. Physica 1985;16(D):285-317.

Kaplan, J.L., Yorke, J.A.. Chaotic behavior of multidimensional difference equations. In: Functional Differential Equations and Approximations of Fixed Points. Berlin: Springer; 1979, p. 204-227.

Leonov, G.A.. Lyapunov functions in the attractors dimension theory. Journal of Applied Mathematics and Mechanics 2012;76(2).

Broer, H.W., Dumortier, F., van Strien, S.J., Takens, F.. Structures in dynamics: finite dimensional deterministic studies. Amsterdam: North-Holland; 1991.

Leonov, G.A.. Strange attractors and classical stability theory. St.Petersburg: St.Petersburg University Press; 2008.

Rossler, O.E.. An equation for continuous chaos. Physics Letters A 1976;57(5):397-398.

Rossler, O.E.. Continuous chaos - four prototype equations. Annals of the New York Academy of Sciences $1979 ; 316(1): 376-392$. 
Pesin, Y.B.. Dimension type characteristics for invariant sets of dynamical systems. In: Russian Mathematical Surveys, 43:4. 1988, p. 111-151. doi:10.1070/RM1988v043n04ABEH001892.

Temam, R.. Infinite-dimensional Dynamical Systems. Springer; 1993.

Boichenko, V.A., Leonov, G.A., Reitmann, V.. Dimension Theory for Ordinary Differential Equations. Stuttgart: Teubner; 2005.

Leonov, G.A., Lyashko, S.A.. Lyapunov's direct method in estimates of the fractal dimension of attractors. Differential Equations 1997;33(1):67-74.

Leonov, G.A.. The upper estimations for the Hausdorff dimension of attractors. Vestnik of the St Petersburg University: Mathematics 1998;(1):19-22.

Boichenko, V.A., Leonov, G.A., Franz, A., Reitmann, V.. Hausdorff and fractal dimension estimates for invariant sets of non-injective maps. Zeitschrift fur Analysis und ihre Anwendung 1998;17(1):207-223.

Boichenko, V.A., Leonov, G.A.. On estimated for dimension of attractors of the Henon map. Vestnik of the St Petersburg University: Mathematics 2000;33(13):8-13.

Leonov, G.A., Reitmann, V., Slepukhin, A.S.. Upper estimates for the Hausdorff dimension of negatively invariant sets of local cocycles. Doklady Mathematics 2011a;84(1):551-554. doi:10.1134/ S1064562411050103.

Leonov, G.A., Pogromsky, A.Y., Starkov, K.E.. Dimension formula for the Lorenz attractor. Physics Letters, Section A: General, Atomic and Solid State Physics 2011b;375(8):1179-1182.

Szczepaniak, A., Macek, W.M.. Unstable manifolds for the hyperchaotic Rossler system. Physics Letters A 2008;372(14):2423-2427. doi:10.1016/j .physleta.2007.12.009.

Li, Q.. A topological horseshoe in the hyperchaotic Rossler attractor. Physics Letters A 2008;372(17):29892994. doi:10.1016/j physleta.2007.11.071.

Golub, G.H., van Loan, C.F.. Matrix Computations. Johns Hopkins University Press; 1996.

Parker, T.S., Chua, L.O.. Practical Numerical Algorithms for Chaotic Systems. Springer-Verlag; 1989.

Oseledec, V.I.. Multiplicative ergodic theorem: Characteristic lyapunov exponents of dynamical systems. In: Transactions of the Moscow Mathematical Society; vol. 19. 1968, p. 179-210.

Yan, G., Ruan, L.. Lattice Boltzmann solver of Rossler equation. Communications in Nonlinear Science and Numerical Simulation 2000;5(2):64-68. doi:10.1016/S1007-5704(00)90003-0.

Al-Sawalha, M.M., Noorani, M.S.M.. Application of the differential transformation method for the solution of the hyperchaotic Rossler system. Communications in Nonlinear Science and Numerical Simulation 2009;14(4):1509-1514. doi:10.1016/j.cnsns. 2008.02.002.

Letellier, C., Elaydi, S., Aguirre, L.A., Alaoui, A.. Difference equations versus differential equations, a possible equivalence for the Rossler system? Physica D: Nonlinear Phenomena 2004;195(1-2):29-49. doi: 10.1016/j.physd. 2004.02.007.

Barrio, R., Blesa, F., Serrano, S.. Qualitative analysis of the Rossler equations: Bifurcations of limit cycles and chaotic attractors. Physica D: Nonlinear Phenomena 2009;238(13):1087-1100. doi:10.1016/j . physd. 2009.03.010. 
Barrio, R., Blesa, F., Serrano, S.. Qualitative and numerical analysis of the Rossler model: Bifurcations of equilibria. Computers and Mathematics with Applications 2011;62(11):4140-4150. doi:10.1016/j . camwa . 2011.09 .064$.

Leonov, G.A., Kuznetsov, N.V., Vagaitsev, V.I.. Localization of hidden Chua's attractors. Physics Letters A 2011c;375(23):2230-2233. doi:10.1016/j.physleta.2011.04.037.

Bragin, V.O., Vagaitsev, V.I., Kuznetsov, N.V., Leonov, G.A.. Algorithms for finding hidden oscillations in nonlinear systems. The Aizerman and Kalman conjectures and Chua's circuits. Journal of Computer and Systems Sciences International 2011;50(4):511-543. doi:10.1134/S106423071104006X.

Leonov, G.A., Kuznetsov, N.V., Vagaitsev, V.I.. Hidden attractor in smooth Chua systems. Physica D 2012;241(18):1482-1486. doi:10.1016/j.physd.2012.05.016.

Leonov G. A. Kuznetsov, N.V.. Hidden attractors in dynamical systems. From hidden oscillations in HilbertKolmogorov, Aizerman, and Kalman problems to hidden chaotic attractors in Chua circuits. International Journal of Bifurcation and Chaos 2013;23(1):1-69. doi:10.1142/S0218127413300024.

\section{Acknowledgements}

This work was supported by the Academy of Finland, Russian Ministry of Education and Science (Federal target programm), Russian Foundation for Basic Research and Saint-Petersburg State University. 


\section{Appendix: Computation of Lyapunov exponents and Lyapunov dimension in MATLAB}

Here it is given the main parts of program, written in MATLAB, which implements the described above algorithm for the computation of Lyapunov dimension of three-dimensional dynamical system (f.e. it is considered Rössler system (1.1)).

Listing 1: Computation of Lyapunov exponents

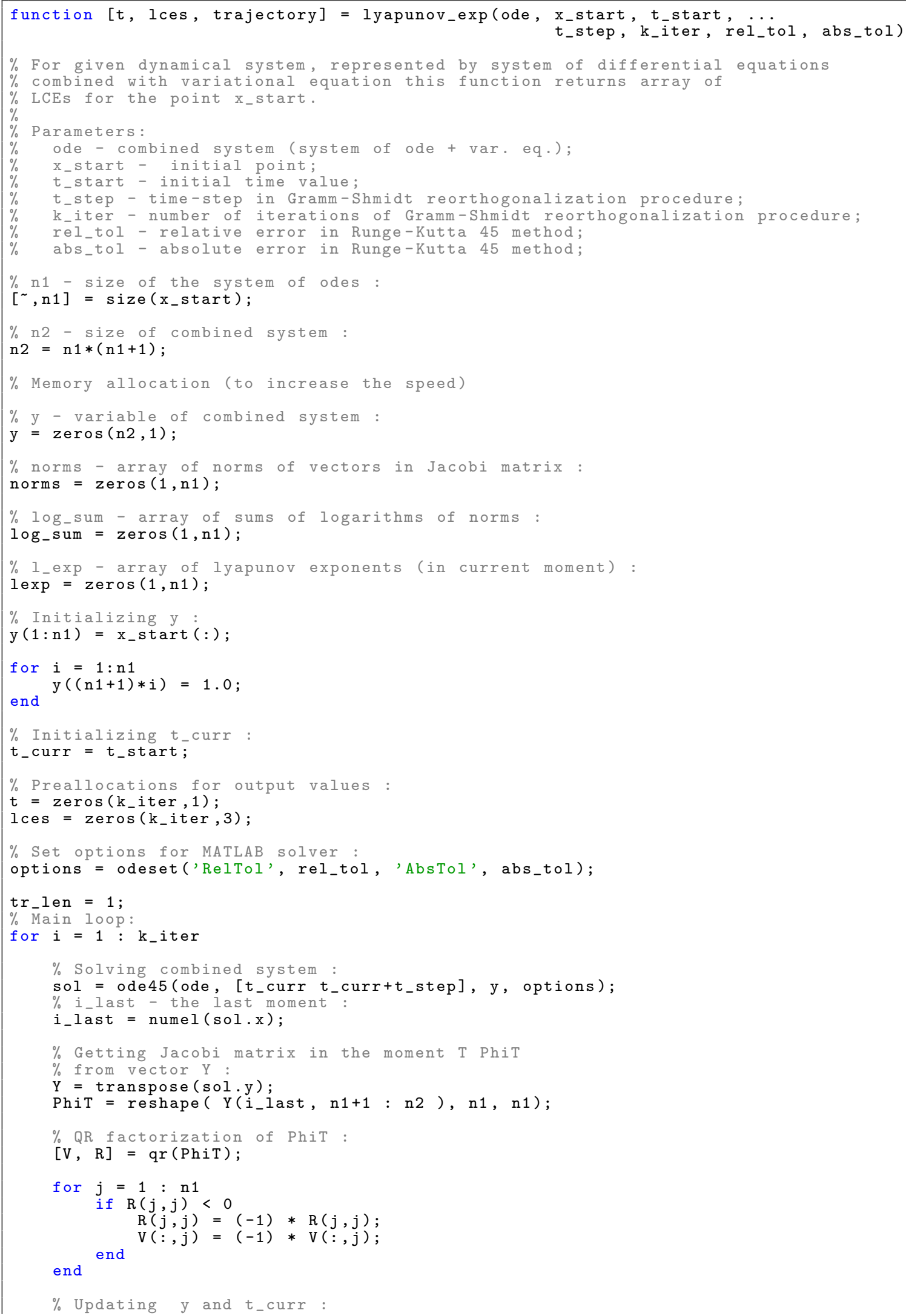




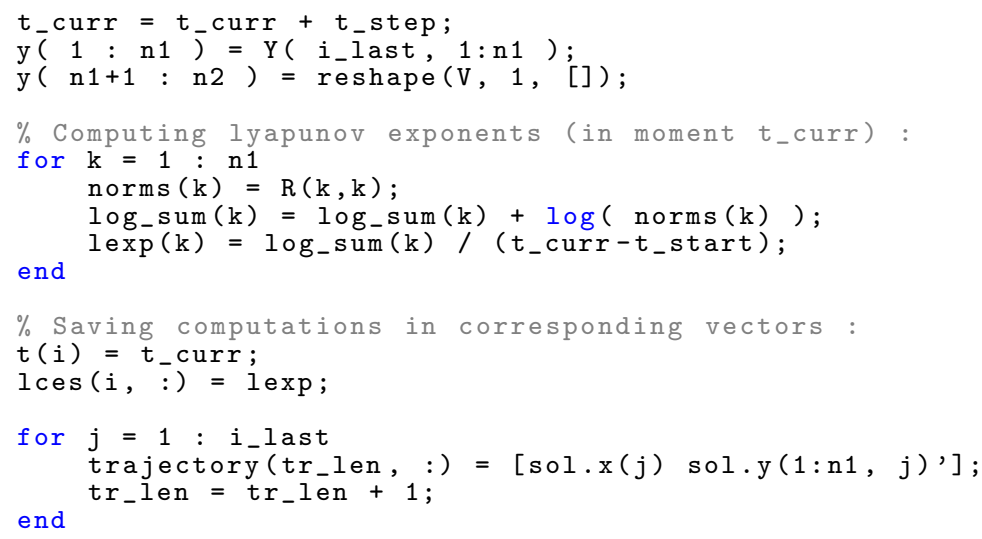

Listing 2: Computation of Lyapunov dimension

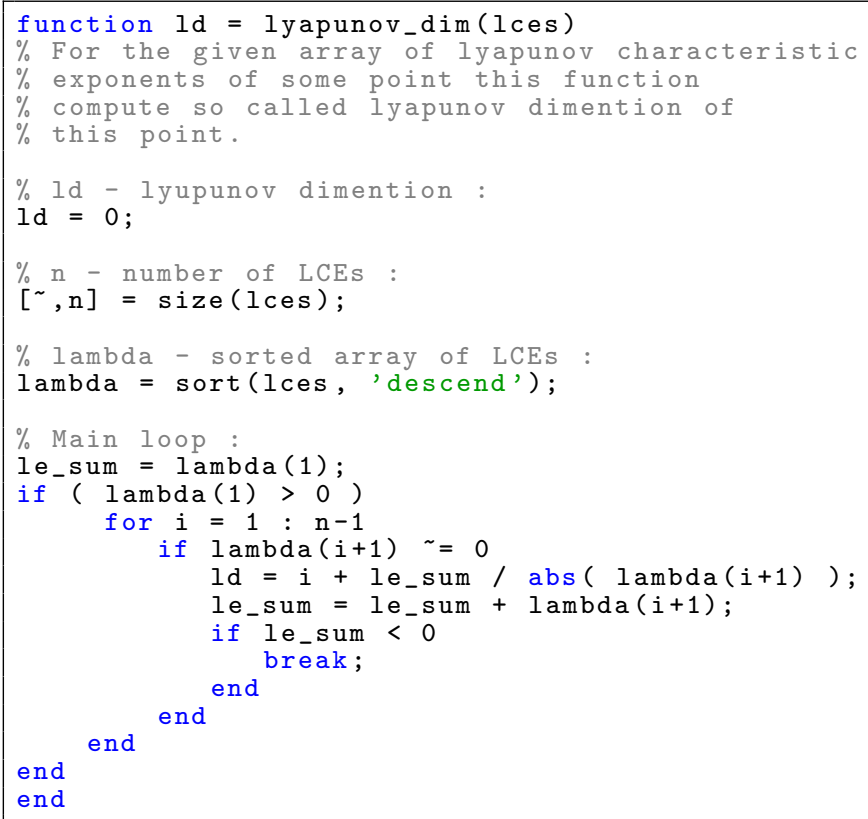

Listing 3: Rössler system (1.1)

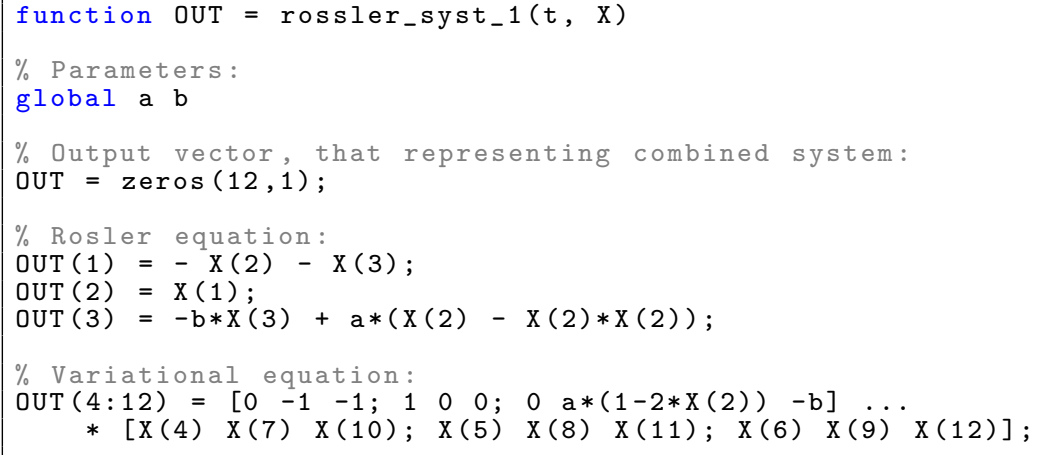

Listing 4: Numerical procedure for Rössler system (1.1)

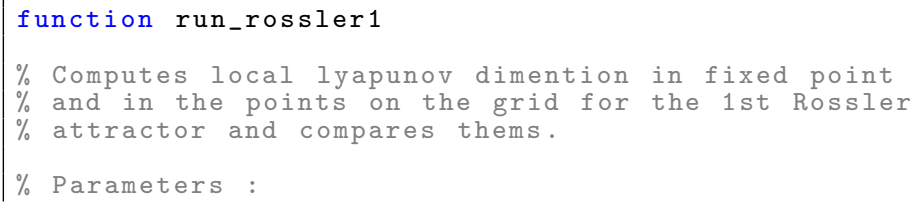




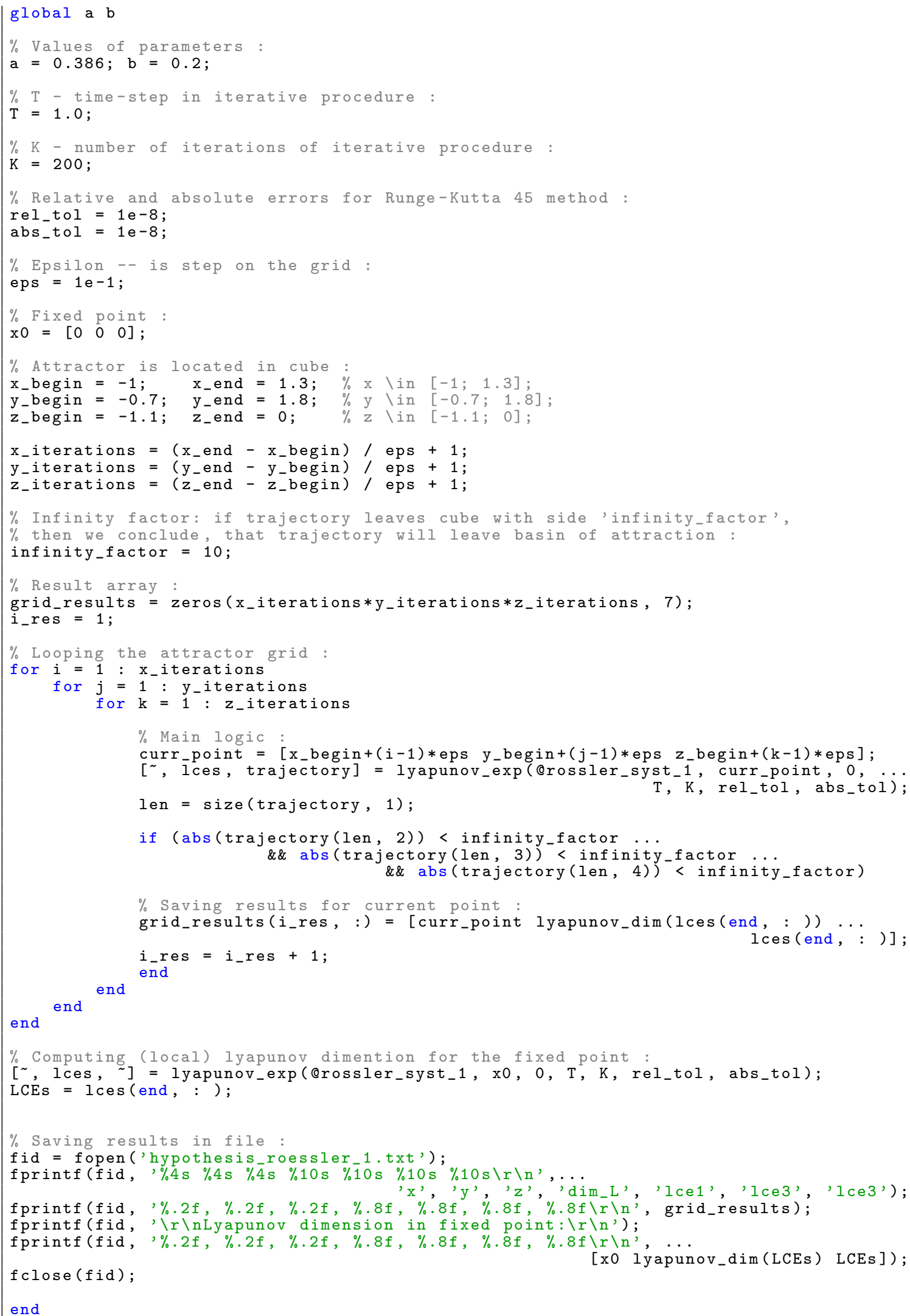

